\title{
La energía del viento en México: Simulación de un parque eólico y aplicación de análisis probabilístico de seguridad
}

\section{Wind Power in Mexico: Simulation of a Wind Farm and Application of Probabilistic Safety Analysis}

\author{
C. Martín del Campo-Márquez \\ Departamento de Sistemas Energéticos, \\ Facultad de Ingeniería, UNAM. \\ E-mail:cecilia.martin.del.campo@gmail.com \\ P.F. Nelson-Edelstein \\ Departamento de Sistemas Energéticos, \\ Facultad de Ingeniería, UNAM. \\ E-mail:pnelson_007@yahoo.com \\ M.Á. García-Vázquez \\ Departamento de Sistemas Energéticos, \\ Facultad de Ingeniería, UNAM. \\ E-mail:maiki27@yahoo.com
}

(Recibido: agosto de 2007; aceptado: marzo de 2008)

\section{Resumen}

Se presentan los aspectos más importantes referentes a la energía eólica en México, su potencial de aprovechamiento y los proyectos planeados. Se comparan sus costos de generación eléctrica con los de otras fuentes de energía. Se presentan los resultados de la simulación con el programa WindPro, de un parque eólico de 100 MW localizado en el Istmo de Tehuantepec. Asimismo, se analizan algunos de los impactos ambientales relacionados con la instalación de parques eólicos en la zona mencionada. Finalmente, se discuten las ventajas que pueden aportar los análisis probabilísticos de seguridad para evaluar los riesgos asociados a eventos que pudieran ocurrir en los parques eólicos, siendo los resultados de este análisis de utilidad para el diseño y mantenimiento de los parques y de los propios aerogeneradores. Específicamente se desarrolló un árbol de eventos con el fin de analizar los posibles escenarios que podrían producirse cuando la velocidad del viento es demasiado alta. También, se desarrollaron árboles de fallas para cada uno de los sistemas de mitigación para determinar la importancia relativa en las secuencias de falla de cada uno de los componentes del aerogenerador, con el objetivo de comprobar la rentabilidad de las mejoras y la optimización del mantenimiento.

Descriptores: Energía del viento, simulación de un parque eólico, análisis probabilístico de seguridad.

\footnotetext{
Abstract

The most important aspects of wind energy in Mexico, including the potential for generating electricity and the major projects planned are presented here. In particular, the generation costs are compared to those of other energy sources. The results from the simulation of a $100 \mathrm{MW}$ wind farm in the Tehuantepec Isthmus are also presented. In addition, the
} 
environmental impacts related to the wind farm in the mentioned zone are analyzed. Finally, some benefits of using Probabilistic Safety Analysis are discussed with respect to evaluating the risks associated with events that can occur in wind parks, being especially useful for design and maintenance of the parks and the wind turbines themselves. In particular, an event tree was developed to analyze possible accident sequences that could occur when the wind speed is too great. Also, fault trees were developed for each mitigating system considered, in order to determine the relative importance of the wind generator components to the failure sequences, in order to evaluate the yield of suggested improvements and the optimization of maintenance programs.

Keywords: Wind power, wind farm simulation, probabilistic safety analysis.

\section{Introducción}

Los costos de generación eléctrica han sido el dato principal para la selección de plantas de generación eléctrica. Recientemente, en los países más desarrollados ya se consideran también los recursos disponibles y los impactos a la salud y al medio ambiente en la toma de decisiones. Ahora es recomendable realizar análisis de sustentabilidad de las diferentes opciones de generación eléctrica para definir las políticas de planeación de la expansión eléctrica.

Los procesos convencionales para generar electricidad que se basan en la quema de combustibles fósiles (principalmente carbón y combustóleo), emiten cantidades importantes de gases de efecto invernadero (GEI) y otros contaminantes atmosféricos. El tema del calentamiento global y sus consecuentes cambios climáticos, ha motivado a buscar tecnologías energéticas no convencionales que emitan cantidades reducidas de GEI. La energía eólica, por tratarse de una energía prácticamente libre de emisiones de GEI, puede contribuir a mitigar el cambio climático, además de diversificar las fuentes de generación de energía eléctrica.

Dentro de las energías renovables, en los últimos años, la energía eólica ha tenido un crecimiento sumamente dinámico a nivel mundial (figura 1), con un crecimiento del 32\% en el año 2006, (Global Wind Energy Council, 2007).

Aunque la participación de la eoloelectricidad es todavía inferior al 1\% de la producción eléctrica mundial, más de 70 países tienen ya instalaciones eólicas y planes de crecimiento. La tabla 1 lista los cinco países en el mundo con la mayor capacidad eólica instalada, (Global Wind Energy Council, 2007).

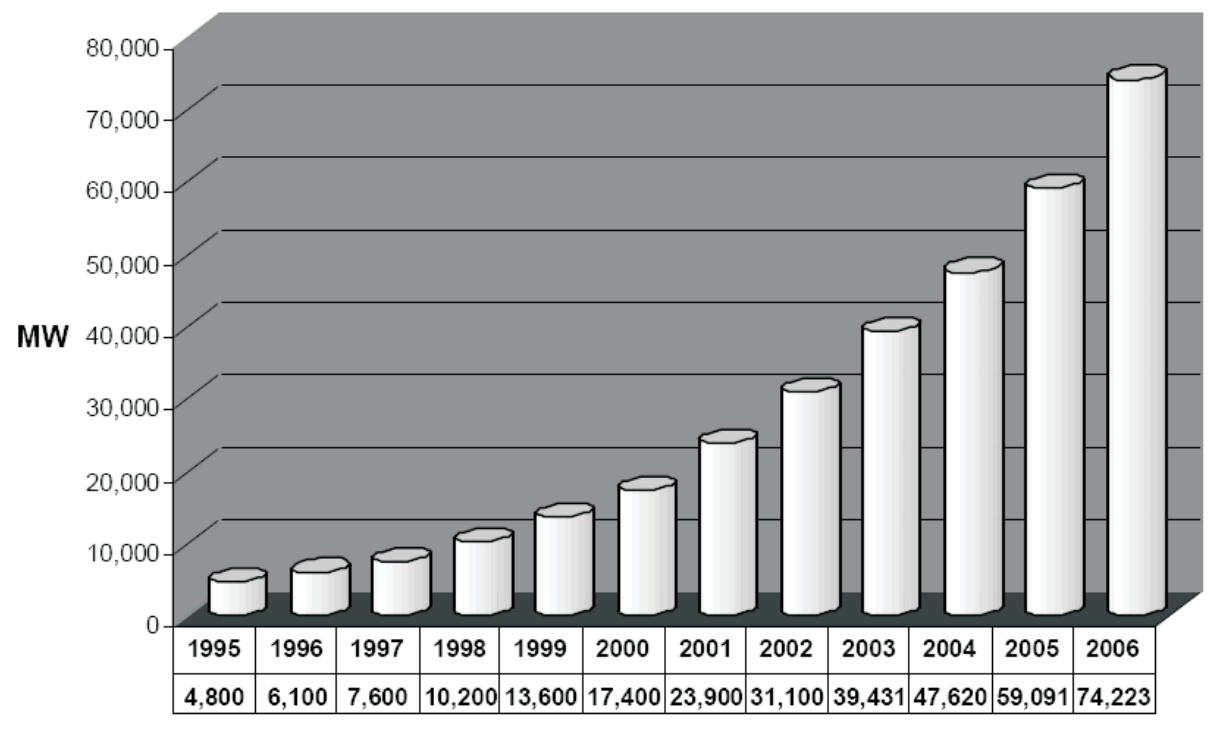

Figura 1. Capacidad Instalada Mundial - Energía Eólica en MW 


\section{La energía eólica en México}

La Comisión Federal de Electricidad (CFE) ha dado a conocer información sobre el potencial eólico estimado en la República Mexicana ${ }^{1}$, el cual se muestra en la tabla 2. Los sitios más estudiados y con el mayor potencial son el Istmo de Tehuantepec y la Península de Baja California.

En 1994, México puso en marcha su primer parque eólico localizado en el ejido de La Venta, en el Istmo de Tehuantepec, Oaxaca. El parque eólico La Venta, construido como proyecto prototipo, fue el primero en su tipo en nuestro país y en América Latina y cuenta con siete aerogeneradores de $225 \mathrm{~kW}$, que han operado con un factor de planta de alrededor de $40 \%$.

En enero de 2007, entró en operación comercial el segundo parque eólico, La Venta II, localizado también en el Istmo de Tehuantepec. Se trata de un proyecto de obra pública financiada, adjudicado en el año 2005 a un consorcio español. Este nuevo parque eólico, cuenta con 98 aerogeneradores de $850 \mathrm{~kW}$ y una capacidad total instalada de 83.3 MW, (Portal CFE). Con esta adición, la capacidad eólica actual es de 85.48 MW de los 48,259.59 MW de la capacidad total instalada por CFE (Portal CFE). Actualmente la CFE contempla en el escenario de planeación (CFE, 2006), cinco proyectos denominados La Venta III, Oaxaca I, II, III y IV con una capacidad de 101.4 MW cada uno, sus características se muestran en la tabla 3.

\section{Simulación de un parque eólico}

En esta sección se presenta la simulación de un parque eólico de $100 \mathrm{MW}$, constituido por 100 aerogeneradores de 1000 kW cada uno; situado en el ejido Santo Domingo en el Istmo de Tehuantepec. La simulación fue desarrollada con la ayuda de los módulos BASIS, PARK, DECIBEL y METEO del software WindPRO (EMD International). Las características del sitio, tales como la rugosidad del terreno y los posibles obstáculos para el viento, fueron detallados en la base de datos del simulador. La figura 2 señala la ubicación aproximada de este parque en la República Mexicana.

Tabla 1. Principales países por capacidad eólica instalada

\begin{tabular}{ccc}
\hline Lugar & País & Capacidad instalada MW \\
\hline 1 & Alemania & 20,621 \\
2 & España & 11,615 \\
3 & Estados Unidos & 11,603 \\
4 & India & 6,270 \\
5 & Dinamarca & 3,136 \\
\hline
\end{tabular}

Tabla 2. Potencial eólico en México

\begin{tabular}{cc}
\hline \multicolumn{2}{c}{ Potencial eólico en México (MW) } \\
\hline Sur del Istmo de Tehuantepec & $2,000-3,000$ \\
Península de Baja California & $1,500-2,500$ \\
Península de Yucatán & $1,000-2,000$ \\
Zacatecas & $800-1,500$ \\
Costa del Pacífico & $1,000-1,500$ \\
Golfo de México & $1,000-1,500$ \\
\hline
\end{tabular}


DOI: http://dx.doi.org/10.22201/fi.25940732e.2009.10n4.031

La energía del viento en México: Simulación de un parque eólico y aplicación de análisis probabilístico de seguridad

Tabla 3. Proyectos eoloeléctricos: Escenario de planeación de CFE

\begin{tabular}{cccccc}
\hline Año & Proyecto & $\begin{array}{c}\text { Capacidad Bruta } \\
\text { MW }\end{array}$ & $\begin{array}{c}\text { Capacidad Neta } \\
\text { MW }\end{array}$ & $\begin{array}{c}\text { Generación Media Anual } \\
\text { (GWh) }\end{array}$ & $\begin{array}{c}\text { Número de } \\
\text { unidades }\end{array}$ \\
\hline 2008 & La Venta III & 101.4 & 99 & 361 & 78 \\
2009 & Oaxaca I & 101.4 & 99 & 373 & 78 \\
2011 & Oaxaca II,III y IV & 304.2 & 297 & 1119 & 234 \\
\hline
\end{tabular}

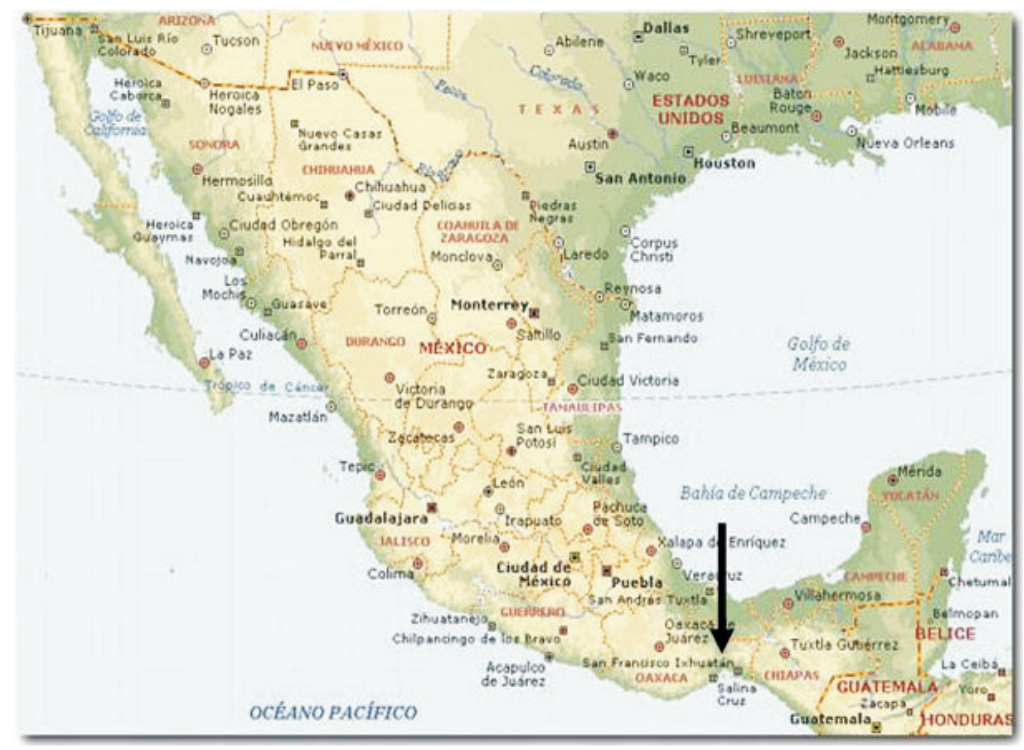

Figura 2. Localización del parque eólico simulado

El arreglo físico de un proyecto eólico, consiste en la distribución de los aerogeneradores en el área destinada para la central. Generalmente, se distribuyen a lo largo de líneas paralelas que forman un ángulo recto con la dirección de los vientos dominantes. La separación entre las líneas de aerogeneradores y la separación entre cada aerogenerador dependen del diámetro del rotor de cada unidad. La separación debe ser calculada para que se minimice la interferencia entre turbinas y las pérdidas por arreglo cuando existen cambios en la dirección del viento.

Una amplia separación de los aerogeneradores producirá más energía, pero a expensas de más superficie terrestre, de más caminos, así como también de más cableado eléctrico. Consecuentemente determinar la distribución de aerogeneradores es un problema de optimización con restricciones, en el que el costo de generación es la variable a minimizar.

En este trabajo, se simuló un parque constituido por 4 filas de 25 aerogeneradores cada una, con un espaciamiento de 3 diámetros de rotor entre aerogeneradores y 6 diámetros de rotor entre las filas de aerogeneradores. Cabe mencionar que el arreglo de aerogeneradores empleado en esta simulación (distancia entre las turbinas) es teóricamente el mínimo recomendado para que el parque tenga un buen desempeño. Conforme a lo anterior, el parque eólico simulado en este trabajo, ocupará aproximadamente un área de 700 hectáreas. Los resultados más importantes de la simulación son los siguientes:

Análisis Energía vs Sector, incluido en el análisis de producción del parque del módulo METEO: calcula la cantidad de energía anual que puede ser obtenida por sector (N, NNE, ENE, E, ESE, SSE, S, SSW, WSW, W, WNW y NNW). El resultado fue que el viento proveniente del sector norte $(\mathrm{N})$; es decir, de Norte a Sur, es ampliamente dominante con una generación anual de $4000 \mathrm{MWh}$ y despreciable para los otros sectores.

Generalmente, los aerogeneradores aprovechan vientos con velocidad de 5 a $25 \mathrm{~m} / \mathrm{s}$. Con menos de 5 
$\mathrm{m} / \mathrm{s}$ los aerogeneradores son muy ineficientes y arriba de $25 \mathrm{~m} / \mathrm{s}$ están diseñados para detener el giro de las aspas, con el objeto de evitar daños en los equipos del aerogenerador. La figura 3 muestra el reporte del análisis de Energía vs Velocidad del Viento, realizado con WinPro, indica la distribución de la energía en $\mathrm{MWh}$ al año, para un rango de velocidades de viento promedio de 0 a 25 $\mathrm{m} / \mathrm{s}$. Se observa que el parque eólico, en el mejor de los casos, podría generar del orden de $475 \mathrm{MWh}$ al año, si la velocidad promedio anual del viento en el sitio fuera de $15 \mathrm{~m} / \mathrm{s}$.

El módulo DECIBEL calcula el nivel de ruido producido por el parque eólico, al que estaría sometido el asentamiento urbano más cercano, Santo Domingo, el cual es de $40.7 \mathrm{~dB}(\mathrm{~A})$. La figura 4 muestra el esquema de la propagación del ruido producido por el parque eólico; se tiene $55 \mathrm{~dB}(\mathrm{~A})$ dentro del parque y valores de 50, 45, 40 y $35 \mathrm{~dB}(\mathrm{~A})$ desde la zona más cercana a la más alejada mostrada en el esquema.

\section{Evaluación económica}

En esta sección se presentan los resultados de cálculos para obtener el costo del $\mathrm{kWh}$ generado por una planta eoloeléctrica. Los costos unitarios de inversión se expresan en dólares por $\mathrm{kW}$ a precios medios del 2005. El cálculo se hizo a moneda constante, considerando una tasa de descuento del 12\%. El análisis se inicia tomando como base el costo instantáneo (overnight), 1,110 $\mathrm{Dls} / \mathrm{kW}$ y un programa de inversión a 12 meses, similar al empleado en el COPAR-Generación (COPAR, 2005). En la tabla 4 se hace un condensado de los datos necesarios para calcular el costo nivelado del $\mathrm{MWh}$ por concepto de inversión, operación y mantenimiento. Con estos datos y mediante el empleo de las ecuaciones para la obtención del costo nivelado del MWh, se hizo la tabla 5 .

Este costo de 44.54 dólares/MWh eólico es competitivo con los de la tecnología nuclear y del ciclo combinado, 43.17 dólares/MWh y 49.57 dólares/MWh, respectivamente (COPAR, 2005).

Ahora bien, se debe tener presente que el viento es intermitente y que en más de la mitad del tiempo durante el año, no se está generando energía y es necesario contar, en algún sitio relativamente cercano al parque eólico, con una potencia de respaldo igual a la capacidad instalada del parque, de alguna fuente energética no intermitente. Esto representa un fuerte inconveniente, debido a la inversión de capital que debe hacerse para contar con este respaldo. Sin embargo, se han planteado algunas soluciones a esta situación mediante la utilización de energía hidroeléctrica que actualmente ya está instalada en la región sur del país, mediante una planeación del despacho de carga de estas hidroeléctricas. También sería factible que parte de los aerogeneradores, bombearan agua en alguna presa cercana, de manera que la hidroeléctrica generará, cuando la eólica no se encuentre disponible por falta de viento; sin embargo, este aspecto queda para estudiarse con más detalle.

\section{Consideraciones ambientales}

Si bien, la migración de las aves es un fenómeno que sucede a nivel mundial, este estudio únicamente se ha basado en lo concerniente a la posible alteración que provocaría un parque eólico en el medio ambiente y en el hábitat de ciertas especies de aves en México.

La figura 5 muestra algunas de las rutas (USGS, 2005) que siguen ciertas especies durante su vuelo al Sur; se puede observar que éstas pasan a través del

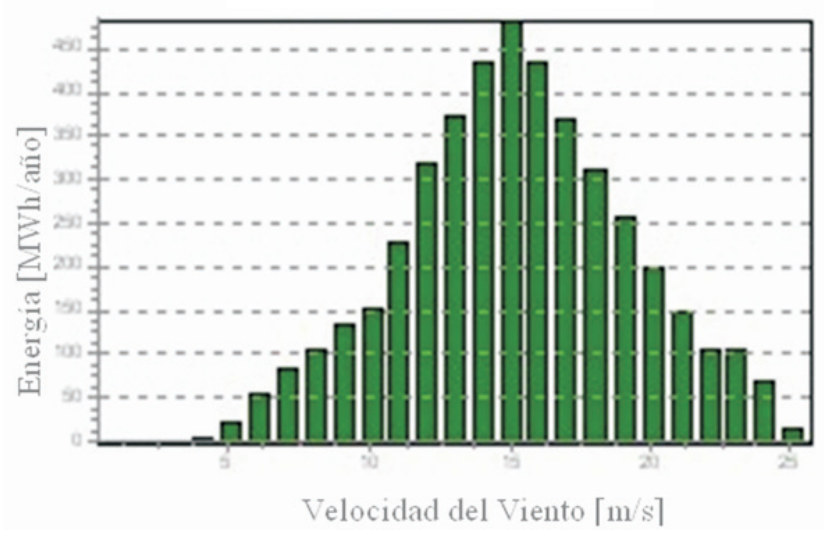

Figura 3. Análisis energía vs velocidad del viento 
territorio nacional, por toda la costa del Golfo de México y prácticamente todo el Istmo de Tehuantepec; que es justamente la zona en la cual se planean instalar los principales proyectos eólicos en México, y donde se encuentra el parque eólico simulado en esta investigación.

Como ya se mencionó, La Venta I cuenta solamente con 7 aerogeneradores, situados relativamente cerca unos de otros; sin embargo, desde 1994, año en que inició su operación, no ha sido documentada la muerte de ave alguna. Ahora, con la puesta en marcha de La Venta II (98 aerogeneradores), tendrá que tomarse en cuenta, el cómo este proyecto y los que están por venir, afecten lo menos posible la vida que llevan las aves del lugar.

Tabla 4. Datos para el cálculo del costo nivelado del MWh

\begin{tabular}{cc}
\hline Potencia efectiva $(C)$ & $1000 \mathrm{~kW}$ \\
Usos propios $($ up $)$ & 0.001 fracción \\
Factor de planta $(f p)$ & 0.439 fracción \\
Costo instantáneo $(C U)$ & $1110 \mathrm{Dol} / \mathrm{kW}$ \\
Tasa de descuento $(i)$ & 0.12 fracción \\
Vida de la planta $(n)$ & 20 años \\
Tiempo Construcción $(N)$ & 1 año \\
\hline
\end{tabular}

Tabla 5. Costo del MWh eólico

\begin{tabular}{cc}
\hline Costo inversión actualizado & $1,182.64 \mathrm{Dol} / \mathrm{kW}$ \\
Costo anual de inversión & $158.33 \mathrm{Dol} / \mathrm{kW}$-año \\
Costo anual de O\&M & $30.0 \mathrm{Dol} / \mathrm{kW}$-año \\
Generación neta anual & $3841.79 \mathrm{horas} /$ año \\
Costo de inversión (nivelado) & $36.73 \mathrm{Dol} / \mathrm{MWh}$ \\
Costo O\&M (nivelado) & $7.81 \mathrm{Dol} / \mathrm{MWh}$ \\
Costo nivelado del $\mathbf{M W h}$ & $\mathbf{4 4 . 5 4} \mathrm{Dol} / \mathbf{M W h}$ \\
\hline
\end{tabular}

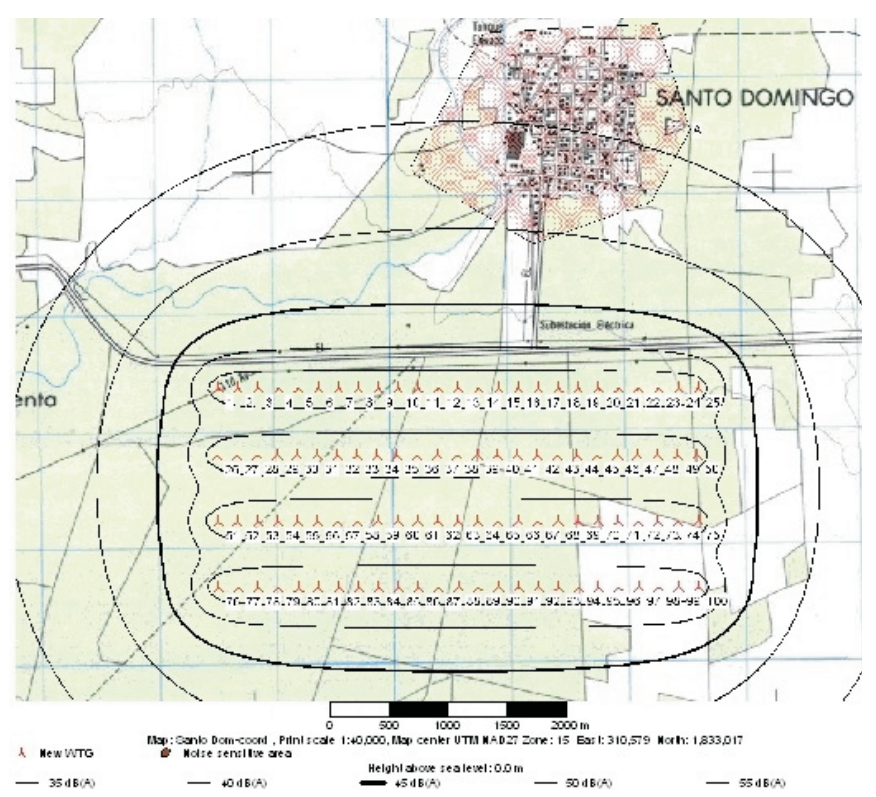

Figura 4. Esquema de la propagación del ruido producido por el parque eólico 


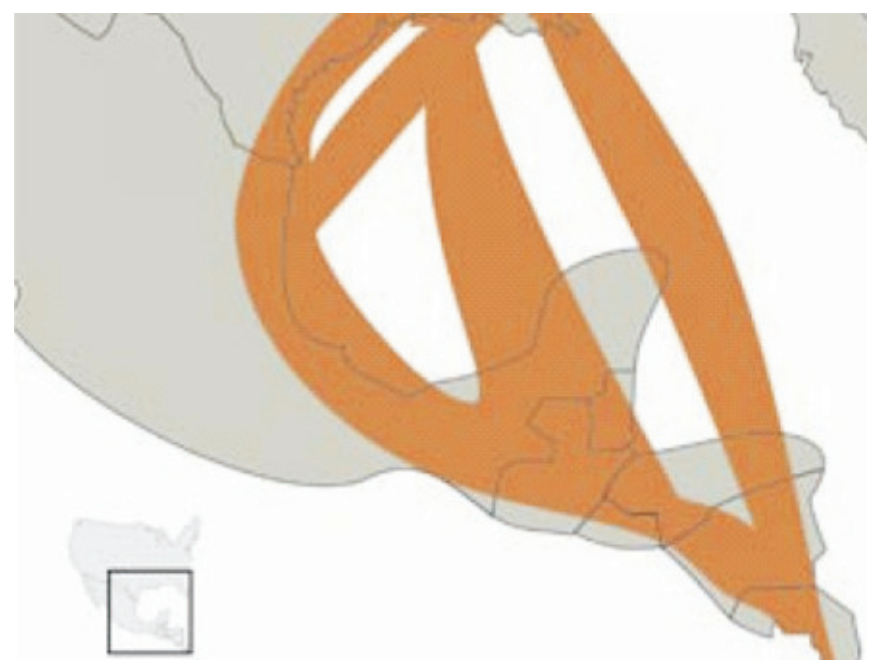

Figura 5. Rutas migratorias a través del istmo de Tehuantepec y el Golfo de México

Por otro lado, cabe mencionar que el ruido producido por los aerogeneradores es otro impacto sobresaliente relacionado con los parques eólicos. En lo referente a este problema, en México será prohibida la construcción de un parque eólico, si el nivel de ruido producido excede el límite máximo establecido en la Norma Oficial Mexicana NOM-081-SEMARNAT (1994), creada para tal propósito por la Secretaría de Salud, tomando en cuenta el ruido máximo permitido para humanos, 65 $\mathrm{dB}(\mathrm{A})$. De acuerdo a los resultados de la simulación, el parque propuesto cumple con la norma.

\section{Análisis probabilístico de seguridad (APS) de parques eólicos}

Si bien, las centrales eoloeléctricas no causan muertes o enfermedad durante el transcurso de su vida útil, se presentan otros riesgos, tales como la ruptura de alguna pala o la expulsión del hielo que se ha formado en las palas del aerogenerador, si es que éste se encuentra situado en una zona de bajas temperaturas, así como riesgos económicos y la posible colisión de embarcaciones contra aerogeneradores, en el caso de ser un parque localizado mar adentro.

Es por esto que organizaciones internacionales han considerado el uso del Análisis Probabilístico de Seguridad (APS) para el estudio de los riesgos pertenecientes a la energía del viento, con el objetivo de comprobar la rentabilidad de las mejoras en los diseños y la optimización del mantenimiento.

En esta sección se muestra un ejemplo de la aplicación de APS, en el cual se desarrolló un árbol de eventos con el fin de analizar los posibles escenarios que podrían producirse cuando la velocidad del viento sobrepasa el límite operacional; así también, se desarrollaron los árboles de fallas para cada uno de los sistemas de mitigación, para determinar la importancia relativa en las secuencias de falla de cada uno de los componentes del aerogenerador.

\section{Descripción del sistema "Aerogenerador"}

Los aerogeneradores de La Venta II están diseñados para mantenerse en pie aún con velocidades de viento de 130 $\mathrm{km} / \mathrm{h}$; sin embargo, cuando la velocidad del viento excede $25 \mathrm{~m} / \mathrm{s}$, el sistema de control debe detener el giro del rotor para evitar daños en los equipos.

A continuación, se describe el comportamiento que podría tener un sistema llamado "Aerogenerador", al presentarse un evento iniciador denominado Velocidad de Viento Excedida (OVERSPEED) que se define como una velocidad superior a $25 \mathrm{~m} / \mathrm{s}$ pero inferior a $36 \mathrm{~m} / \mathrm{s}$ (equivalente a los $130 \mathrm{~km} / \mathrm{h}$ ). Para esto, se empleó el programa SAPHIRE, que es una herramienta desarrollada para el Análisis de Confiabilidad y Riesgo. En dicho programa se modeló el sistema "Aerogenerador"; para el cual se dibujaron y cuantificaron el árbol de eventos y los árboles de fallas para cada uno de los subsistemas que conforman este sistema. En total, son cinco los subsistemas básicos en los que se dividió al sistema "Aerogenerador": Sistema de Orientación y Medición (SOYM), Sistema de Control (SC), Operador (OP), Operador Remoto (OPR) y Frenos de Pala (SF). 
DOI: http://dx.doi.org/10.22201/fi.25940732e.2009.10n4.031

La energía del viento en México: Simulación de un parque eólico y aplicación de análisis probabilístico de seguridad

\section{Árbol de eventos}

El trabajo con APS se inició con el desarrollo de un árbol de eventos que considera la posibilidad de velocidades de viento que no superan los $36 \mathrm{~m} / \mathrm{s}$, pero que exceden los $25 \mathrm{~m} / \mathrm{s}$. Esto implicaría que el aerogenerador debe detenerse; si esto no sucede, se tiene el riesgo de que se dañe. Para lo anterior, se cuenta con los denominados "sistemas de mitigación", mismos que aparecen en el encabezado del árbol de eventos.

La figura 6 muestra el árbol de eventos desarrollado en SAPHIRE. Se pueden observar las 12 secuencias que se generaron en correspondencia al comportamiento de los subsistemas y a la relación entre ellos, al presentarse el evento iniciador "Overspeed". De estas 12 secuencias, sólo siete tienen como estado final "falla" y es en ellas en las que se enfoca este análisis.

A continuación, a modo de ejemplo, se detalla la secuencia número 7; es decir, se indica el orden en el que deben presentarse las fallas para que el sistema pueda llegar al estado final "falla". Esta se señala en color azul en la figura 6.

Secuencia 7: Se presenta viento entre 25 y $36 \mathrm{~m} / \mathrm{s}$. El anemómetro lo detecta. El sistema de control falla. El operador en el parque no se percata de lo ocurrido y el operador remoto tampoco se da cuenta del problema. El aerogenerador se daña.

\section{Reporte de medidas de importancia}

En esta sección se presenta el reporte de medidas de importancia (tabla 6); el cual enlista los eventos representativos del modelo construido. En este caso, se destaca la columna de la reducción de riesgo, la cual clasifica a los componentes de acuerdo a su posibilidad de reducir el riesgo; entre más grande sea el valor de un componente, mayor será la importancia del mismo en la reducción de riesgo del sistema. Esta medida de importancia, indica qué tanto puede reducirse la frecuencia de las secuencias, si la probabilidad de falla del componente fuera reducida a cero. Por ejemplo, si el valor que se muestra en dicha tabla para el sistema FRENOS (cuya probabilidad de falla es de $4.0 \times 10^{-2}$ ) fuera reducido a 0.0 resultaría en una reducción del riesgo de $2.885 \times 10^{-2}$.

También se puede observar que el sistema FRENOS (SF) es el más importante, después del evento iniciador (OVERSPEED), cuando se toma como punto de partida la reducción de riesgo, ya que éste presenta el valor más alto entre el conjunto de eventos que aparecen en la tabla. Esto indica que si fuera posible tener unos frenos perfectos que no fallaran, es decir, si se reduce la probabilidad de falla de 4.0E-002 a 0.0, la frecuencia de falla del turbogenerador se reduce de $2.888 \times 10^{-2}$ a $3.0 \times 10^{-5}$ (que se calcula como: $2.888 \times 10^{-2}-2.885 \times 10^{-2}$ ). Con esta información se podría calcular el costo de la reducción de frecuencia de falla del turbogenerador, a través de la reducción de la probabilidad de falla de cada uno de los subsistemas, y así calcular el costo/beneficio para cada inversión. Sea al mejorar el subsistema, optimizar su mantenimiento o implantar programas para reducir los errores humanos.

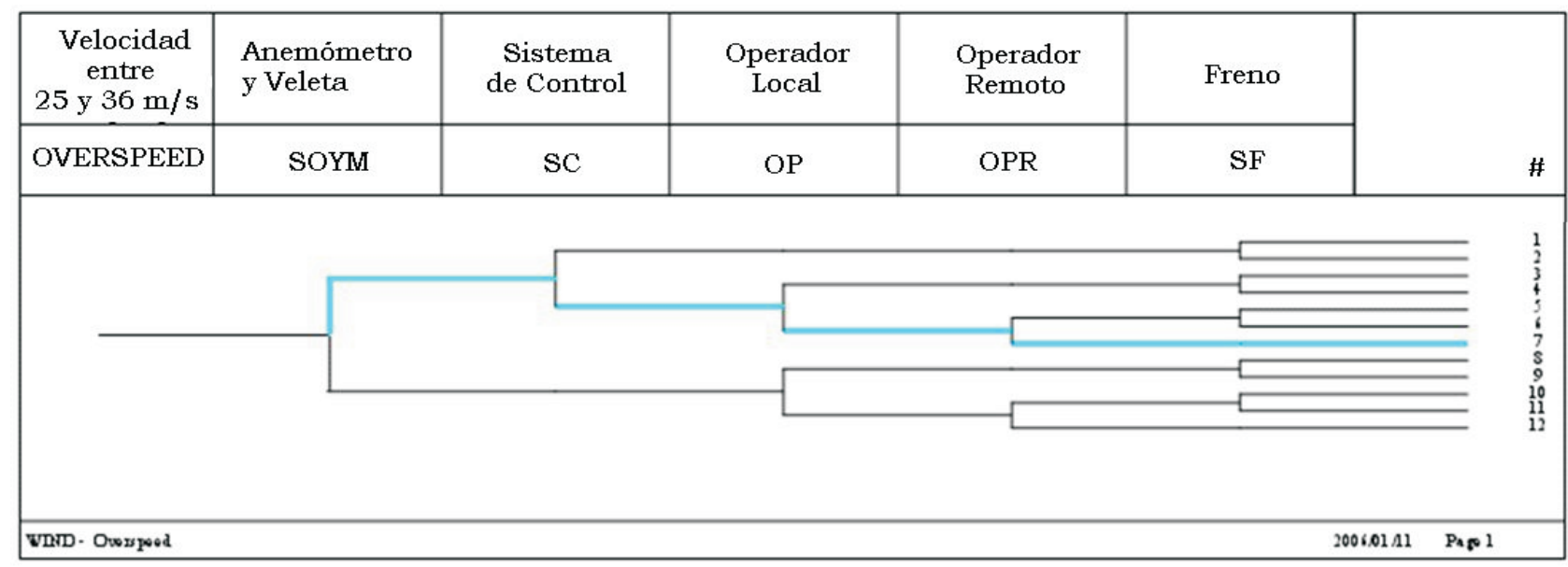

Figura 6. Árbol de eventos para el evento iniciador OVERSPEED 
DOI: http://dx.doi.org/10.22201/fi.25940732e.2009.10n4.031

C. Martín del Campo-Márquez, P.F. Nelson-Edelstein y M.Á. García-Vázquez

Tabla 6. Reporte de medidas de importancia

Ordenado por Intervalo de Reducción de Riesgo

\begin{tabular}{lccc}
\hline \multicolumn{1}{c}{ Nombre del evento } & $\begin{array}{c}\text { Número de } \\
\text { Ocurrencias }\end{array}$ & $\begin{array}{c}\text { Probabilidad de } \\
\text { falla }\end{array}$ & Reducción de Riesgo \\
\hline OVERSPEED (Velocidad excedida) & 17 & $7.000 \mathrm{E}-001$ & $2.888 \mathrm{E}-002^{*}$ \\
FRENOS & 9 & $4.000 \mathrm{E}-002$ & $2.885 \mathrm{E}-002$ \\
CONTROLADOR & 4 & $2.900 \mathrm{E}-002$ & $8.365 \mathrm{E}-004$ \\
CONEXIÓN & 4 & $1.000 \mathrm{E}-003$ & $2.881 \mathrm{E}-005$ \\
SOFTWARE & 4 & $1.000 \mathrm{E}-003$ & $2.124 \mathrm{E}-005$ \\
SUMINISTRO ELÉCTRICO & 4 & $3.000 \mathrm{E}-004$ & $8.644 \mathrm{E}-006$ \\
OPERADOR & 8 & $3.000 \mathrm{E}-003$ & $4.461 \mathrm{E}-006$ \\
OPERADOR REMOTO & 4 & $3.000 \mathrm{E}-002$ & $1.912 \mathrm{E}-006$ \\
ANEMÓMETRO & 4 & $5.000 \mathrm{E}-005$ & $1.442 \mathrm{E}-006$ \\
\hline
\end{tabular}

* Este es el valor de reducción de riesgo total que se puede tener

Asociado a lo anterior y debido a la falta de datos reales; se debe tener presente que para este estudio, los ejercicios realizados han sido desarrollados empleando valores supuestos de las probabilidades, por lo que éstos solamente pueden ser tomados como ensayos.

\section{Trabajo a futuro con APS en parques eólicos}

Si bien, lo que se ha realizado en este estudio es solamente una introducción de lo útil que puede ser la utilización del Análisis Probabilístico de Seguridad en el estudio de parques eólicos; se debe seguir trabajando en la identificación de posibles modos de falla, así como en el desarrollo de los respectivos árboles de eventos para nuevos eventos iniciadores. Además, se debe recopilar información suficiente con el fin de poder desarrollar un análisis cuantitativo que represente la realidad en eventos iniciadores en los que se presente viento con una velocidad superior a la considerada en esta aplicación del APS. Por ejemplo, se podrían analizar eventos, como el del 2 de diciembre del 2006, en el que un aerogenerador de la Venta II, diseñado para soportar velocidades de viento de hasta $130 \mathrm{~km} / \mathrm{h}$, fue derribado ${ }^{2}$ cuando se registraron ráfagas de viento arriba de $190 \mathrm{~km} / \mathrm{h}$. Para este análisis sería necesario disponer de escenarios de vientos, incluyendo las velocidades de viento y sus frecuencias para el sitio del parque eólico, así como la

2 http://www.eluniversal.com.mx/estados/vi_63207.html información sobre la fragilidad estructural de los aerogeneradores para poder calcular la probabilidad de su derrumbamiento, cuando se presenta viento con velocidad superior a la de diseño.

\section{Conclusiones}

La aportación a la planeación de proyectos eólicos por parte del software WindPRO es muy importante, ya que permite predecir el comportamiento que tendrán, tanto el parque, como los aerogeneradores que lo conforman. Esto ayudará a mejorar el desempeño del mismo, reduciendo así, los costos de generación para ese parque en específico.

El costo nivelado del $\mathrm{kWh}$ para los parques eólicos es actualmente competitivo con las fuentes convencionales a pesar de que se trata de unidades generadoras de bajo factor de planta. Sin embargo, dada la intermitencia del viento, es necesario contar con una capacidad de respaldo igual a la potencia del parque de alguna fuente energética no intermitente en algún sitio relativamente cercano al parque.

Los resultados obtenidos mediante el Análisis Probabilístico de Seguridad (APS) en aerogeneradores son de carácter ilustrativo, pues ha sido desarrollado con datos no reales, debido a la carencia de los mismos. Aun así, resulta claro lo importante que puede ser la información obtenida mediante el APS; por lo que es necesaria la obtención de los datos reales de la probabilidad de falla de 
DOI: http://dx.doi.org/10.22201/fi.25940732e.2009.10n4.031

La energía del viento en México: Simulación de un parque eólico y aplicación de análisis probabilístico de seguridad

cada uno de los componentes que conforman al aerogenerador; para de esta forma, conocer a detalle cuál o cuáles son los elementos del sistema que con mayor frecuencia sufren descomposturas, perjudicando el desempeño del aerogenerador y del parque en general.

\section{Referencias}

CFE. Programa de obras e inversiones del sector eléctrico (POISE) 2007-2016. México. 2006.

COPAR. Costos y parámetros de referencia para la formulación de proyectos de inversión. Generación 2005. CFE, Subdirección de Programación. Gerencia de Evaluación y Programación de Inversiones. México. 2005.

EMD International A/S (EMD). WindPro [en línea]. Disponible en: http://www.emd.dk/WindPRO/Frontpage

Global Wind Energy Council-Press Release. Global Wind
Energy Markets Continue to Boom-2006 Another Record Year Industry Delivered 32\% of annual. Bruselas, 2007. Portal Comisión Federal de Electricidad [en línea]. Disponible en: www.cfe.gob.mx

Secretaría del Medio Ambiente. Norma Oficial Mexicana NOM-081-SEMARNAT-1994, Contaminación por Ruido [en línea]. Disponible en:

http://www.genomaf.com/normatividad_semarnat.html SAPHIRE (Systems Analysis Programs for Hands-on Integrated Reliability Evaluations) Version 6.77. Idaho National Engineering and Environmental Laboratory. Division of System Technology Office, Nuclear Regulatory Research, U.S Nuclear Regulatory Commission, Washington D.C.

USGS. Science for a Changing World. Migratory Bird Pathways and the Gulf of Mexico. National Wetlands Research Center, Mayo 2005 [en línea]. Disponible en: http://www.nwrc.usgs.gov/factshts/2005-3069.pdf

\section{Semblanza de los autores}

Cecilia Martín del Campo-Márquez. Es ingeniera en energía por la Universidad Autónoma Metropolitana, ingeniera nuclear por el Instituto Nacional de Ciencias y Técnicas Nucleares de Francia y doctora con mención honorífica en ingeniería nuclear por la Universidad de París XI. Trabajó en el Instituto Nacional de Investigaciones Nucleares y en el Instituto de Investigaciones Eléctricas. Actualmente es profesora de la Facultad de Ingeniería, UNAM. Es líder de la línea de desarrollo "Estudios de expansión del sector eléctrico" y ha sido responsable de varios proyectos de investigación y de vinculación. Es miembro del subcomité académico del campo de conocimiento de energía del Programa de Posgrado en Ingeniería de la UNAM. Tiene nivel 1 del Sistema Nacional de Investigadores. Destaca su colaboración con la Academia de Ingeniería de México en la comisión de especialidad de ingeniería nuclear, como secretaria en su consejo directivo (2006-2008) y en su comité editorial (2008-2010). Su actividad en la Sociedad Nuclear Mexicana ha sido muy intensa, siendo actualmente vicepresidenta/presidenta electa. También es miembro del Consejo Consultivo del Instituto de Ciencia y Tecnología del Distrito Federal y es miembro fundador de la Red Nacional del Hidrógeno. A nivel internacional, es miembro de Women in Nuclear Global, de la American Nuclear Society (ANS) y de la Sección Latinoamericana de la ANS y actualmente, es miembro del Comité Ejecutivo de la División de Ciencias Ambientales de la ANS.

Pamela Fran Nelson-Edelstein. Realizó sus estudios de licenciatura en la Universidad de California, Los Angeles, donde se tituló como ingeniera nuclear en 1982. Posteriormente, realizó una maestría en ingeniería nuclear en 1985 en la misma universidad. Desde 2001, trabaja en la Universidad Nacional Autónoma de México y actualmente es técnico académico de tiempo completo en la Facultad de Ingeniería. Es líder del grupo de trabajo de la línea de desarrollo: "Análisis de Riesgo" y ha sido responsable de varios proyectos de investigación y vinculación. Es tutora de maestría del Programa de Posgrado en Ingeniería de la UNAM. Es miembro fundadora de la Red Nacional del Hidrógeno y de la Sociedad Nuclear Mexicana y actualmente, funge como vice-presidente del comité de gestión de riesgo nuclear en el ASME.

Miguel Ángel García-Vázquez. Es ingeniero eléctrico-electrónico de la Universidad Nacional Autónoma de México. Obtuvo el grado de maestro en ingeniería en energía con mención honorífica por parte la misma institución. Ha participado en dos congresos internacionales con proyectos de investigación referentes al desarrollo y explotación del potencial eólico con el que cuenta México. Actualmente labora para ABB México en la división de robótica, en el área de Vision \& Sensing, participando activamente en proyectos de automatización de procesos. 